\title{
Monitoring of rainfall by ground-based passive microwave systems: models, measurements and applications
}

\author{
F. S. Marzano ${ }^{1,2}$, D. Cimini ${ }^{2}$, and R. Ware ${ }^{3}$ \\ ${ }^{1}$ Dipartimento di Ingegneria Elettronica Università di Roma "La Sapienza", Rome, Italy \\ ${ }^{2}$ Centro di Eccellenza CETEMPS, Università dell'Aquila, L'Aquila, Italy \\ ${ }^{3}$ Radiometrics Corp. and UCAR, Boulder, Colorado, USA
}

Received: 17 November 2004 - Revised: 28 May 2005 - Accepted: 6 June 2005 - Published: 22 July 2005

\begin{abstract}
A large set of ground-based multi-frequency microwave radiometric simulations and measurements during different precipitation regimes are analysed. Simulations are performed for a set of frequencies from 22 to $60 \mathrm{GHz}$, representing the channels currently available on an operational ground-based radiometric system. Results are illustrated in terms of comparisons between measurements and model data in order to show that the observed radiometric signatures can be attributed to rainfall scattering and absorption. An inversion algorithm has been developed, basing on the simulated data, to retrieve rain rate from passive radiometric observations. As a validation of the approach, we have analyzed radiometric measurements during rain events occurred in Boulder, Colorado, and at the Atmospheric Radiation Measurement (ARM) Program's Southern Great Plains (SGP) site in Lamont, Oklahoma, USA, comparing rain rate estimates with available simultaneous rain gauge data.
\end{abstract}

\section{Introduction}

Ground-based microwave radiometry has been mainly investigated for estimating temperature, water vapor and cloud liquid profiles in the absence of precipitation (Westwater, 1996). However, the increasing use of multi-frequency radiometers in ground-based stations has raised the question of their potential for retrieving also rainfall rate from ground (Marzano et al., 1999; Liu et al., 2001; Marzano et al., 2002). From an experimental point of view, one of the main problems of ground-based radiometry for rainfall retrieval is the possible impact of water layers on the receiving antenna whose measurements can be heavily contaminated (Jacobson et al., 1986). From a modelling point of view, the approach to rainfall signature characterization requests a thorough insight into the electromagnetic interaction between the microwave radiation and the scattering medium. The radiative transfer theory has been so far the most used approach to take into ac-

Correspondence to: F. S. Marzano

(marzano@die.uniroma1.it) count multiple scattering and vertical inhomogeneity of the atmosphere in the presence of hydrometeor scattering (Tsang et al., 1985).

Following previous works (Marzano et al., 1999; Marzano et al., 2002), the objective of this paper is to investigate about the rainfall signature on multispectral microwave measurements from ground. We develop inversion algorithms for ground-based retrieval of surface rainrate, adopting a modelbased approach. For both stratiform and convective precipitation, we use a radiative transfer model (RTM), including spherical liquid, melt and ice hydrometeors. From the solution of the forward models, by varying the model parameters in a physically-based manner, it is derived a synthetic database made by the downwelling simulated brightness temperatures, the associated mean radiative temperature and the total path-attenuation. Non-linear statistical inversion algorithms are developed for different sets of frequency channels, observation geometries, cloud types, and precipitation intensities. A special care is devoted to set up robust estimators in presence of a random noise which can be attributed to various sources, first of all effects of possible water-films on the antenna reflector. A maximum a posteriori probability discrimination technique is applied to separate stratiform from convective raining clouds.

Simulation results are shown to illustrate the potential of the proposed models by selecting a set of frequencies from 22 to $60 \mathrm{GHz}$, representing the channels currently available on a operational ground-based radiometric system. The latter instrument has been recently upgraded to minimize the effects of hydrometeors on the radiometric measurements, a circumstance experimentally proved as well. As a validation of the approach, we have analyzed rain events that occurred in Boulder, Colorado and at the Atmospheric Radiation Measurement (ARM) Program's Southern Great Plains (SGP) site. Results are illustrated in terms of comparisons between measurements and model data in order to show that the observed radiometric signatures can be attributed to rainfall scattering and absorption. Rain rate estimates are also compared with available simultaneous rain gauge data. 

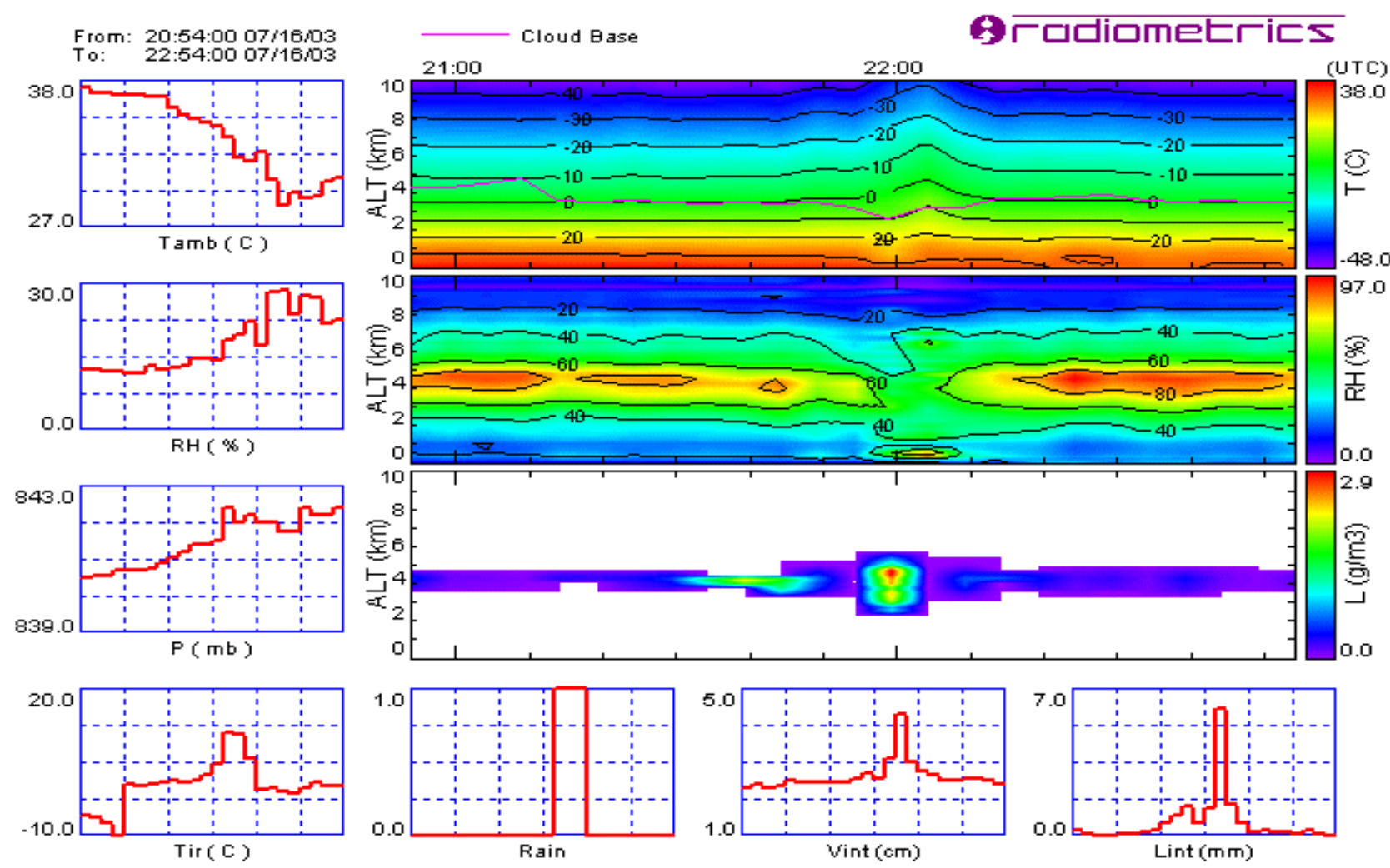

Fig. 1. An example of MWRP operational screen output. Plots refer to data collected during 16 July 2003, in Boulder, Colorado, USA. A rain shower happened roughly at 22:00 UTC. Surface temperature, relative humidity, and pressure are shown on the left. Infrared cloud temperature, rain detection, integrated content of vapour and liquid are shown in the bottom. Contour plots show time-height cross sections of atmospheric temperature (top), relative humidity (middle) and liquid water (bottom).

\section{Radiometric modeling of rainfall}

A general theoretical framework to model the down-welling brightness temperature, measured by a microwave radiometer and due to clouds and precipitation, is given by the radiative transfer theory. Consider a stratified atmosphere with a bottom level at $z=0$ (surface) and a top height at $z=H$. Let us define a vertical coordinate in terms of optical thickness $\tau_{v}$ at frequency $v$ such that $\tau_{\nu}=0$ at $z=H$ and $\tau_{\nu}=\tau_{\nu} \mathrm{s}=\tau_{\nu}(0, \mathrm{H})$ at $z=0$ with $k e_{v}$ the extinction coefficient $\left[\mathrm{km}^{-1}\right]$. It is also convenient to introduce the zenith-angle cosine $\mu=|\cos (\theta)|$ with $\theta$ the zenith angle and $0 \leq \theta \leq \pi / 2$ so that $0 \leq \mu \leq 1$ for downward directions and $-1 \leq \mu \leq 0$ for upward directions. For a plane-parallel geometry, the unpolarized azimuthallysymmetric down-welling brightness temperature $T_{b}\left(\tau_{v} s \mu\right)$, observed from ground at a frequency $v$, can be formally expressed by means of the integral form of radiative transfer equation (RTE), that is:

$T_{b}\left(\tau_{\nu s}, \mu\right)=T_{b}(0, \mu) e^{-\tau_{\nu s} / \mu}+\frac{1}{\mu} \int_{0}^{\tau_{\nu s}} J\left(\tau_{\nu}, \mu\right) e^{-\left(\tau_{\nu s}-\tau_{\nu}\right) / \mu} d \tau_{v}$ where $J\left(\tau_{\nu} \mu\right)$ is the pseudo source function given by:

$$
J\left(\tau_{\nu}, \mu\right)=\frac{w}{2} \int_{-1}^{1} p\left(\mu, \mu^{\prime}\right) T_{b}\left(\tau_{v}, \mu^{\prime}\right) d \mu^{\prime}+[1-w] T\left(\tau_{v}\right)
$$

with $w$ the volumetric albedo, $p$ the volumetric azimuthallyaveraged scattering phase function, and $T$ the physical temperature. The first term of $J$ is sometimes referred to as a multiple scattering source, while the second tem represents thermal emission. The discrete-ordinate numerical method has been chosen to numerically solve RTE in this work (Smith et al., 2002). The atmosphere is assumed to consist of $L$ adjacent homogeneous layers in which volumetric albedo $w$, extinction coefficient ke and phase function $\mathrm{p}$ are taken to be constant within layers.

\subsection{Rainfall models}

In previous works we described a technique to use cloudresolving model outputs to physically constrain the vertical correlation of hydrometeor contents within cloud layers. The gross vertical distribution of four species of hydrometeors is described: cloud droplets, raindrops, graupel particles, and snow particles (D'Auria et al., 1998). Cloud structures have 

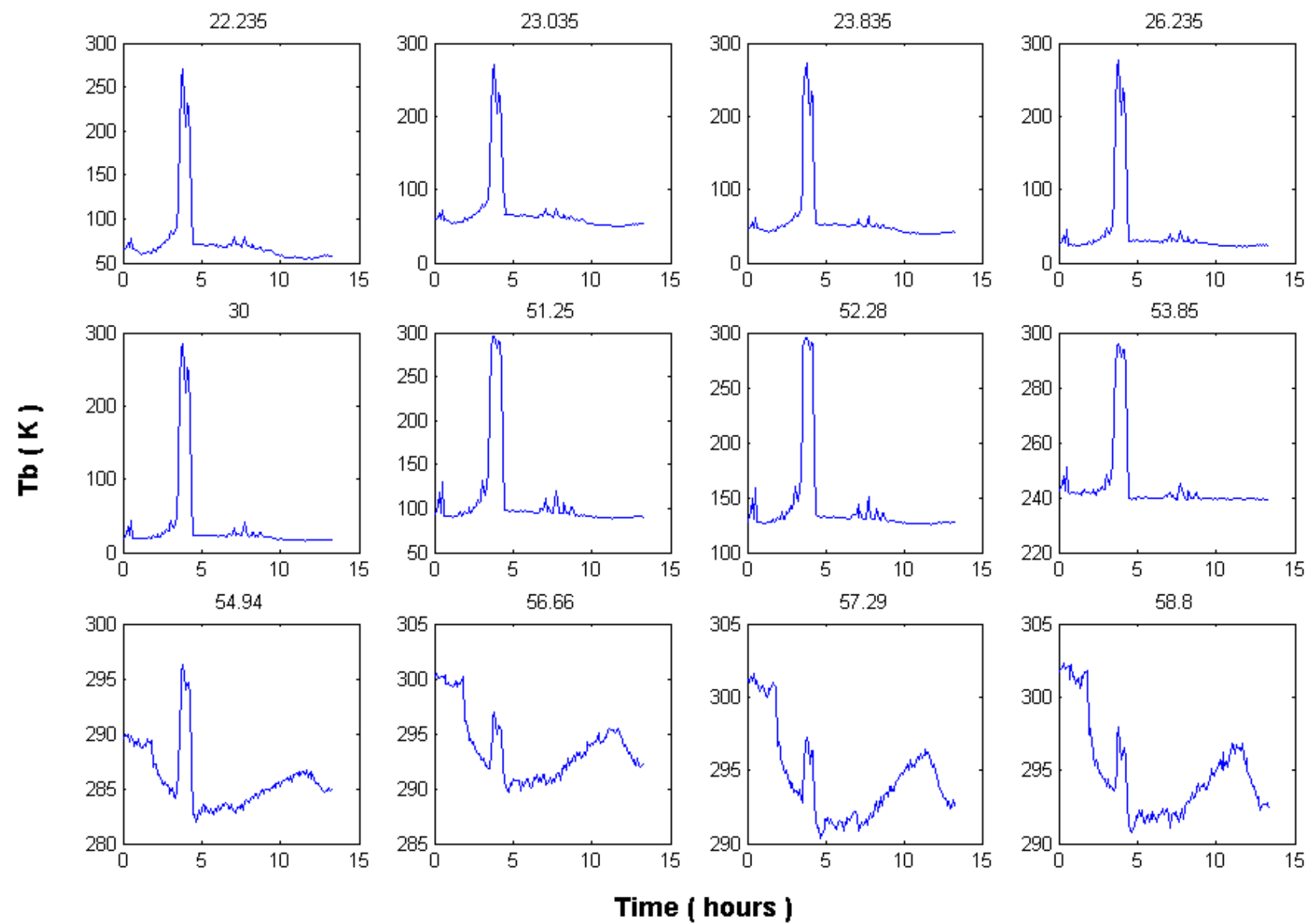

Time ( hours)

Fig. 2. Time series of $\mathrm{Tb}$ observed by the MWRP twelve channels during 17 July 2003 in Boulder, Colorado. A rain shower occurred at 04:00 (elapsed time). For each channel, the corresponding frequency is shown on top $(\mathrm{GHz})$.

been vertically resolved in seven homogeneous layers with a variable vertical resolution of about $1.5 \mathrm{~km}$. The cloud data set, classified into stratiform and convective clouds, has been then extended by means of a Monte Carlo statistical procedure, based on the use of a truncated Gaussian multivariate generator defined by mean and covariance matrix of the hydrometeor contents of each class. Meteorological variables have been supposed to be uniformly variable around their mean values within a given percentage.

In this work we have considered both stratiform rainfall (i.e., nimbostratus) and convective rainfall (i.e., cumulonimbus). Consistently with the RTE adopted scheme, within each cloud layer the temperature has been assumed linearly dependent on the height. Microwave gaseous absorption has been computed by means of the Liebe model. The landsurface emission has been characterized by a Lambertian emissivity model, depending on surface humidity. The humidity value has been supposed randomly variable in order to cover a large variety of surface conditions. The hydrometeor shapes have been assumed all spherical and characterized by inverse-exponential particle size distributions (PSD's). In the logarithmic plane the intercept of a PSD has been derived from the assigned EWC within each layer, while the slope has been parameterized to surface rain-rate using a MarshallPalmer, a Sekhon-Srivastava and a Gunn-Marshall PSD for raindrops, ice graupel and snow, respectively. Cloud droplets have been assumed to follow a modified Gamma PSD. Indeed, oblateness of raindrops can cause a depolarization signal, depending on cloud stage and wind circulation. Even though ice crystals are not spherical, it should be noted that for ground-based rainfall observations the contribution of the iced layers to the Tb's is fairly small (Marzano et al., 1999).

\subsection{Numerical simulations}

The modelling framework does not allow us to take into account the horizontal inhomogeneity of precipitation, that is beam filling problem which might be relevant for convective storm clouds. However, in this respect it has been shown that the one-dimensional (1-D) RTE can be used to approximate three-dimensional (3-D) simulation in the frequency and rainfall ranges considered here by selecting a suitable inclined plane-parallel structure along the line-ofsight (Marzano et al., 1999). By using the coupled rainfall and radiative transfer model illustrated before, a large data set, consisting of 5000 cloud structures together with related 

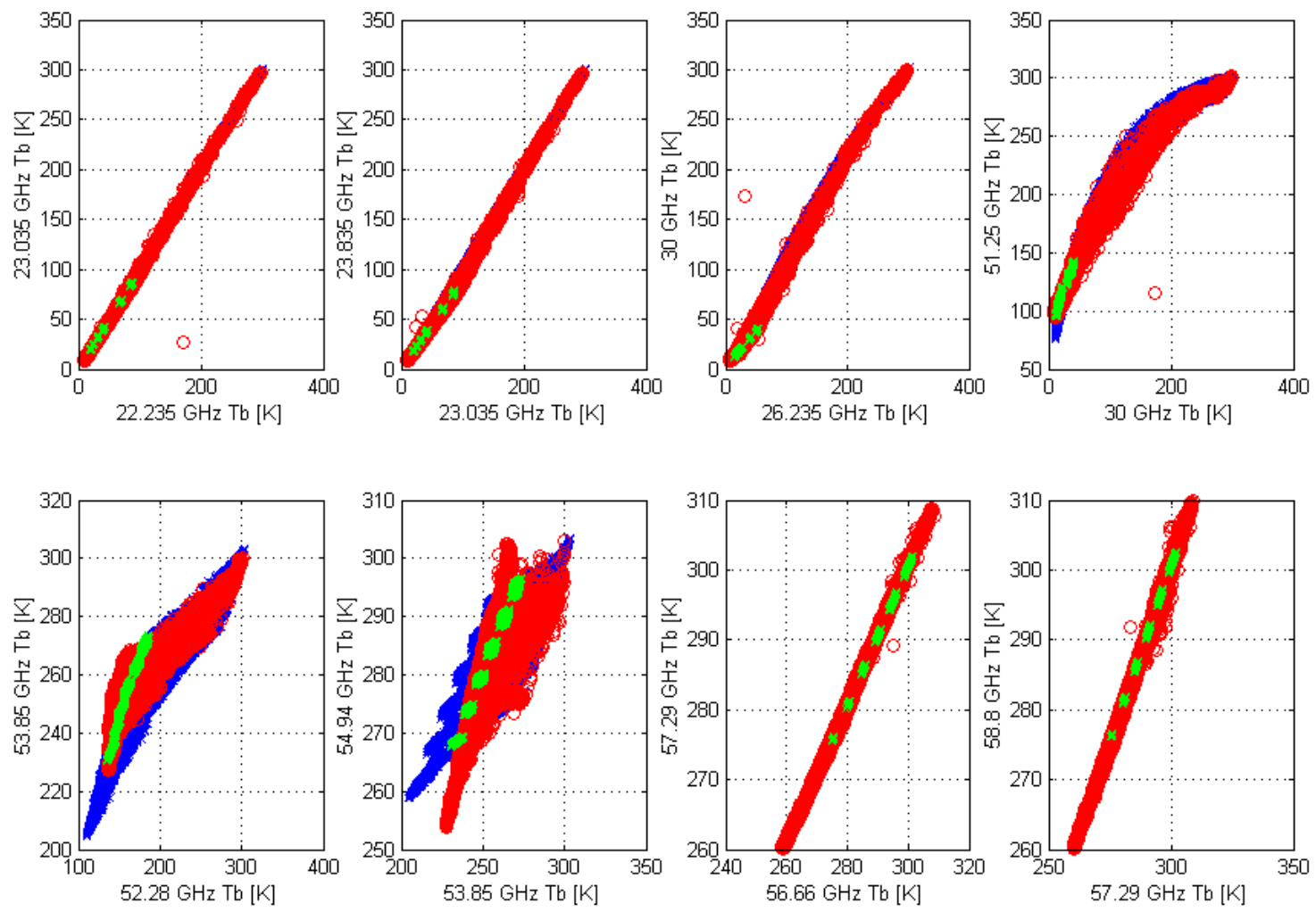

Fig. 3. Rainfall spectral signature in terms of scatterplots between $\mathrm{Tb}$ at different frequencies. About one year of measurements are shown in red. Simulated data set are shown in blue, with non-raining cases evidenced in green.

brightness temperatures at given frequencies and observation angles, has been simulated.

The analysis has included the frequency bands of the operational multi-channel radiometer MWRP. The observation angle has been chosen in accordance to the application, in principle between 0 and 90 degrees elevation. We will show results only for zenith observations in order to be able to compare simulations with radiometric and rain gauge measurements.

\section{Comparison with measurements}

In order to interpret and test the rainfall model simulations, we have used measurements from the Microwave Radiometer Profiler (MWRP), manufactured by Radiometrics Corp. The MWRP can provide, by using neural network inversion algorithms, temperature and humidity soundings up to $10 \mathrm{~km}$ height and low resolution cloud liquid soundings (Ware et al., 2003). The radiometer observes radiation intensity at 12 frequencies in a region of the microwave spectrum that is dominated by atmospheric water vapor, cloud liquid water, and molecular oxygen emissions. The observation frequencies (i.e., 22.035, 22.235, 23.835, 26.235, 30.00, 51.250, 52.280, $53.850,54.940,56.660,57.290,58.800 \mathrm{GHz})$ were chosen by eigenvalue analysis to optimise profile retrieval accuracy. The radiometric profiler includes a vertical infrared sensor and surface temperature, humidity, pressure and rain sensors.
Figure 1 shows the operational real-time output printed on the screen by the proprietary MWRP data acquisition and processing software (VizMet). Time series of temperature, relative humidity, and pressure at the instrument level are shown on the left. Time series of infrared cloud temperature, rain detection, integrated content of vapour and liquid are shown in the bottom. Contour plots show time-height cross sections of atmospheric temperature (top), relative humidity (middle) and liquid water (bottom) retrievals. Thus, the operational output allows the user to monitor in real-time ten fields of meteorological interest simultaneously.

Figure 2 shows the time series of radiometric observation during a rain event that occurred on 17 July 2003 in Boulder, Colorado. Rain occurrence is strongly related to the decrease of temperature, the increase of humidity and the increase of measured Tb's in the window region and saturation/depression in the absorbed band. This behaviour is due to the predominance of either emission or scattering in the $\mathrm{Tb}$ signature from ground with respect to the clear-air value.

Figure 3 shows rainfall spectral signature in terms of scatterplots between $\mathrm{Tb}$ at different frequencies. About one year of radiometric observations at ARM SGP site are involved in this plot. Tb's from the simulated dataset are shown in blue, while measurements are shown in red. Non-raining cases from the simulated database are evidenced in green, thus illustrating the emission and scattering signature mechanisms. 

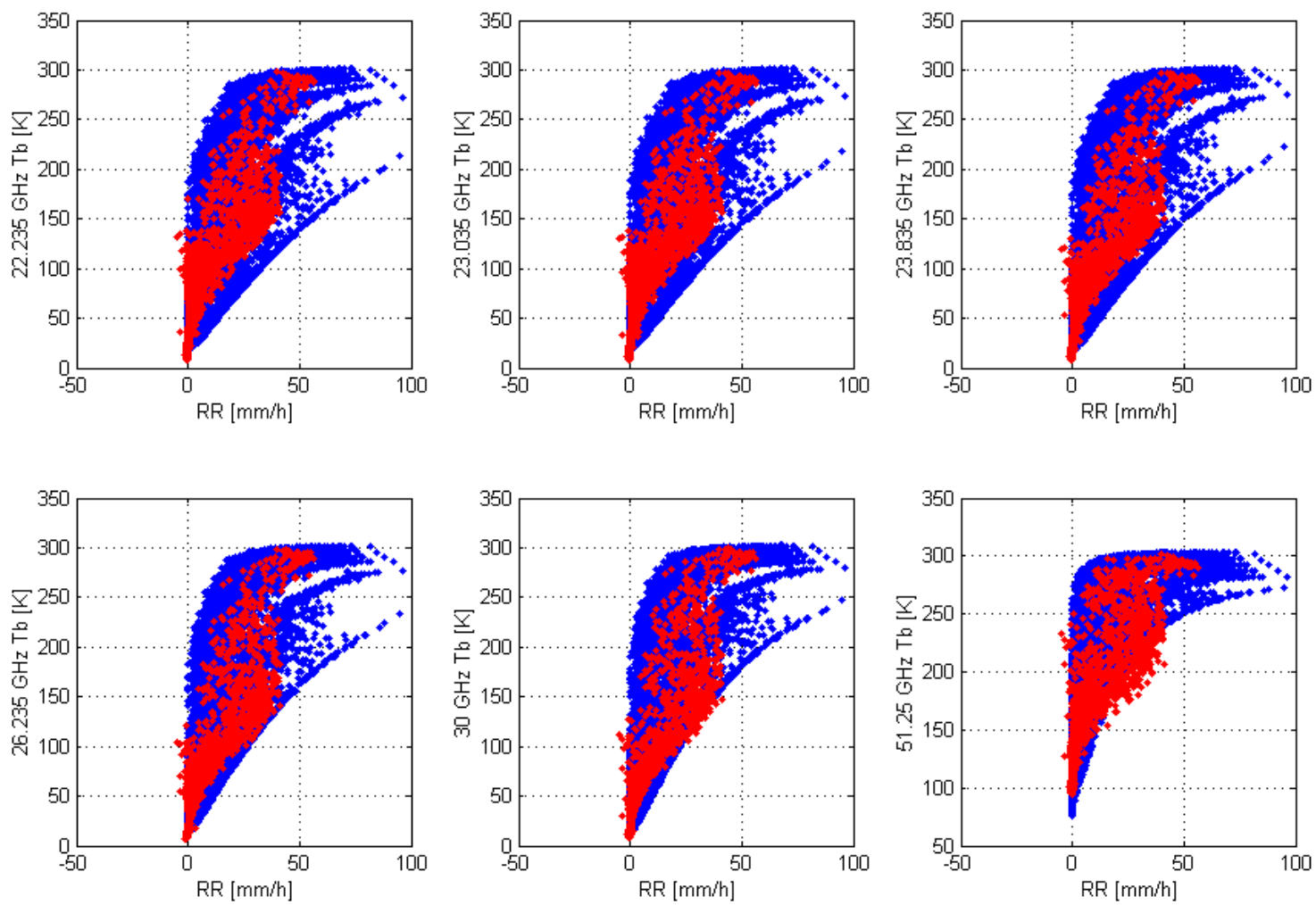

Fig. 4. Scatterplots between $\mathrm{Tb}$ and rain rate. Simulations are shown in blue, while radiometric observations and respective rain rate estimates are shown in red.
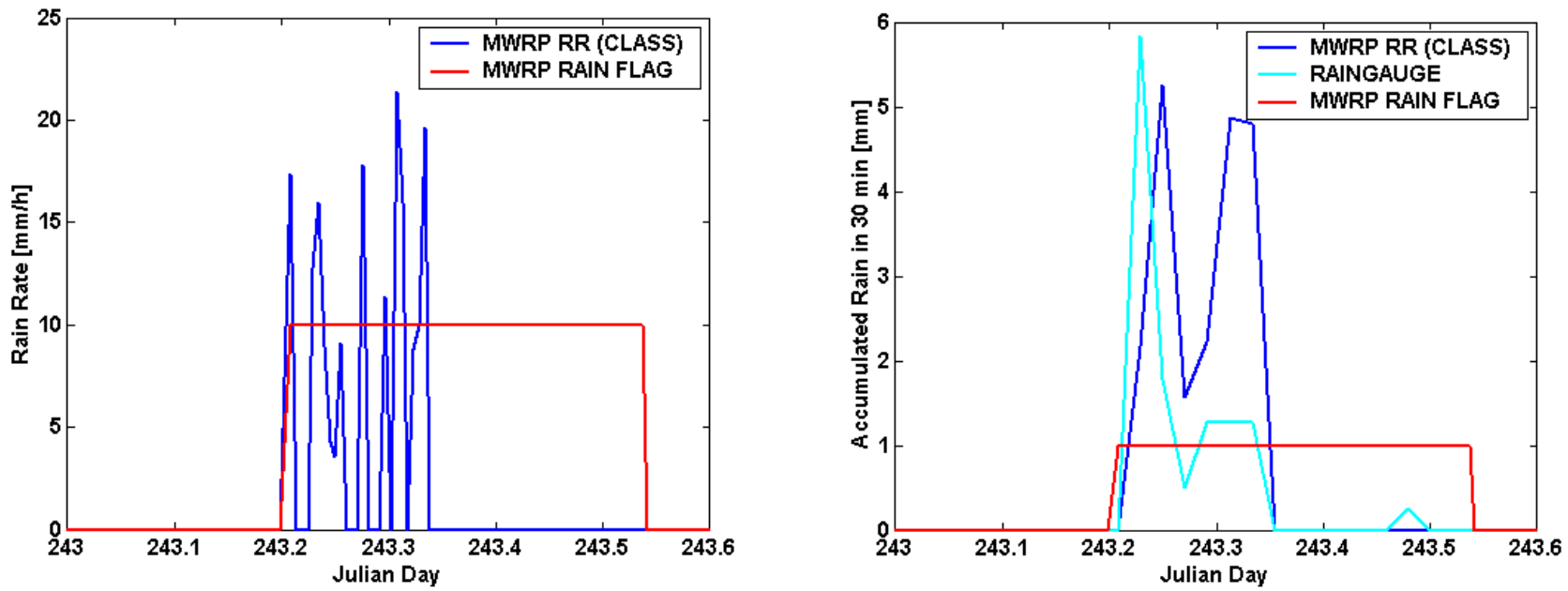

Fig. 5. Time series of MWRP estimated rain rate. Red line, representing rain sensor detection (0/1), has been multiplied by 10 for convenience. Right: Time series of 30-minute accumulated rain for the same case. Red line represents rain sensor detection (0/1). Cyan line represents measurements from a rain gauge, while the blue line represents radiometric estimates.

Figure 3 demonstrate that simulations are able to represent the dynamics of measured $\mathrm{Tb}$ 's, both for weak absorption $(20-30 \mathrm{GHz})$ and strong absorption $(56-60 \mathrm{GHz})$ frequencies. In the $50-55 \mathrm{GHz}$ range there are some differences, which might be related to water film on the antenna or to modelling assumptions. Radiometric simulations have been in a way tuned to the ambient conditions of the observed event. This means that the surface temperature, pressure and humidity have been used to constrain the random meteorological vertical profiles based on standard vertical gradients. 


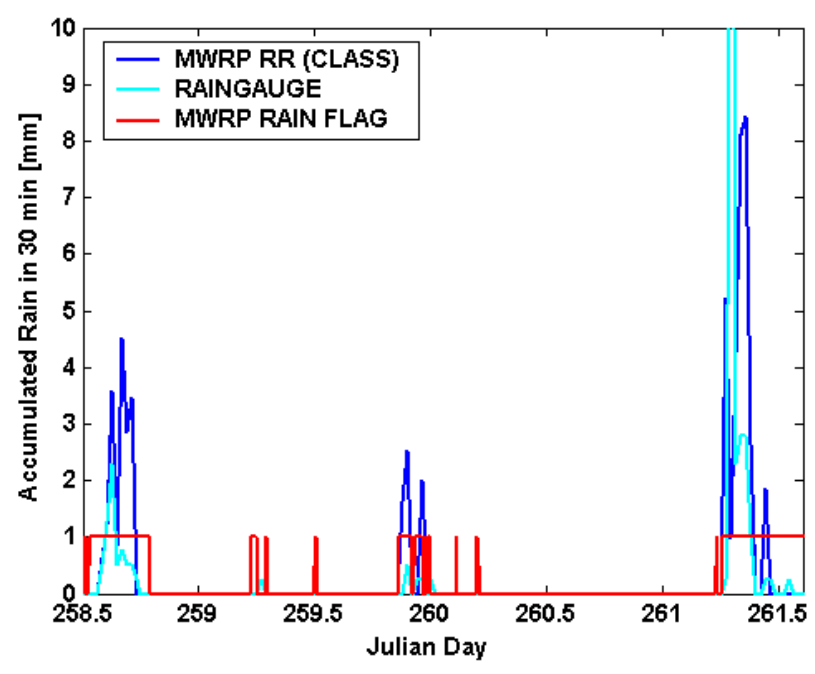

Fig. 6. Same as Fig. 5. More rain events are shown.

Figure 4 shows the relationship between rain rate and $\mathrm{Tb}$ in the weak absorption region $(20-50 \mathrm{GHz})$. Blue dots represent $\mathrm{Tb}$ and rain rate extracted from the simulated database, while red dots correspond to Tb measured by the MWRP and rain rate estimates obtained from radiometric measurements applying a non-linear regression technique. Therefore, Fig. 3 verifies the consistency between measurements and simulations, while Fig. 4 between measurements, simulations and estimates. In the left box of Fig. 5, a time series of rain rate as derived from MWRP observations is plotted in blue. The red line represents the rain sensor detection, which can only be 0 (no rain detected) or 1 (rain detected), although we multiply values by 10 for enhancing the comparison. It is evident that the estimate based on radiometric observations senses rain for a shorter period than does the rain detector. This is due to a residual water film on top of the MWRP, which is misinterpreted by the rain detector. Indeed, the retrieval algorithm we have developed is able to interpret this water as a residual film and distinguish it by actual rainfall.

Right box of Fig. 5 validates this explanation, by comparing measurements from a collocated rain gauge. MWRP rain rates have been averaged into 30-min accumulated rain (blue line) in order to match the rain gauge (cyan line) time resolution, while rain detector measurements are shown in red with their original values (0/1). Rain gauge measurements clearly confirm the duration of the rain shower sensed by radiometric observations.

Similar to Fig. 5, a time series of rain rate at ARM SGP central facility extending for three days is plotted in Fig. 6 . By comparing with rain gauge measurements, it is evident that radiometric estimates follow quite well both the detection of rain and the values of rain rates, although the latter seem to be overestimated. This effect might be related to water film accumulating over the antenna during the rain event. A way to avoid this problem is already under study together with the MWRP manufacturers. Indeed, a development unit, provided with a hydrophobic radome coating, is under testing at the Radiometrics facility.
Preliminary data are shown in Fig. 7, together with observations from an operational unit. The effects of the hydrophobic coating are evident for all of the twelve channels, leaving unchanged non-raining observations, but significantly reducing $\mathrm{Tb}$ during rain. $\mathrm{Tb}$ differences range from about $60 \mathrm{~K}$ at $22.2 \mathrm{GHz}$ channel to about $4 \mathrm{~K}$ at $58.8 \mathrm{GHz}$ channel. Further investigation is needed to understand whether this reduction is sufficient for our purposes. In summary, these preliminary results are quite encouraging; they clearly demonstrate the potential of ground-based radiometry to measure rainfall parameters.

\section{Conclusions and future developments}

We have analysed a large set of ground-based multifrequency radiometric measurements and simulations for different precipitation regimes. The modelled frequencies have been selected in order to match the set of channels currently available on an operational ground-based radiometric system. Rain events occurred in Boulder, Colorado and at the ARM SGP site have been analysed in terms of comparisons between measurements and model data. This comparison has in a way validated that the observed radiometric signatures can be attributed to rainfall scattering and absorption. Moreover, we applied a non-linear inversion technique to estimate rain rate from radiometric observations. Preliminary results are presented, comparing time series of radiometric estimates with rain sensor detections and rain gauge measurements. These results are encouraging, and show the potential of such approach. On the other hand, it shows that the considered radiometric system is not too much affected by water films over the antenna. This can open the use of ground-based multi-frequency microwave radiometry for rain monitoring.

Future developments will regard both modelling and empirical issues. Indeed, the solution of the forward problem might be improved by considering melting layer and depolarisation effects, while the quality of observations would increase if the formation of water film on the antenna is prevented. Finally, further work will be devoted to the analysis of a large set of rainfall cases, and to the comparison with other rain sensors, such as weather radars.

Acknowledgements. This work has been funded by the EU Fifth-Framework EURAINSAT project (EVG2-2000-00522). Support by the Italian Space Agency (ASI), Ministry of Education and Research (MIUR) and by the GNDCI-CNR project is also acknowledged.

Edited by: L. Ferraris

Reviewed by: anonymous referees 

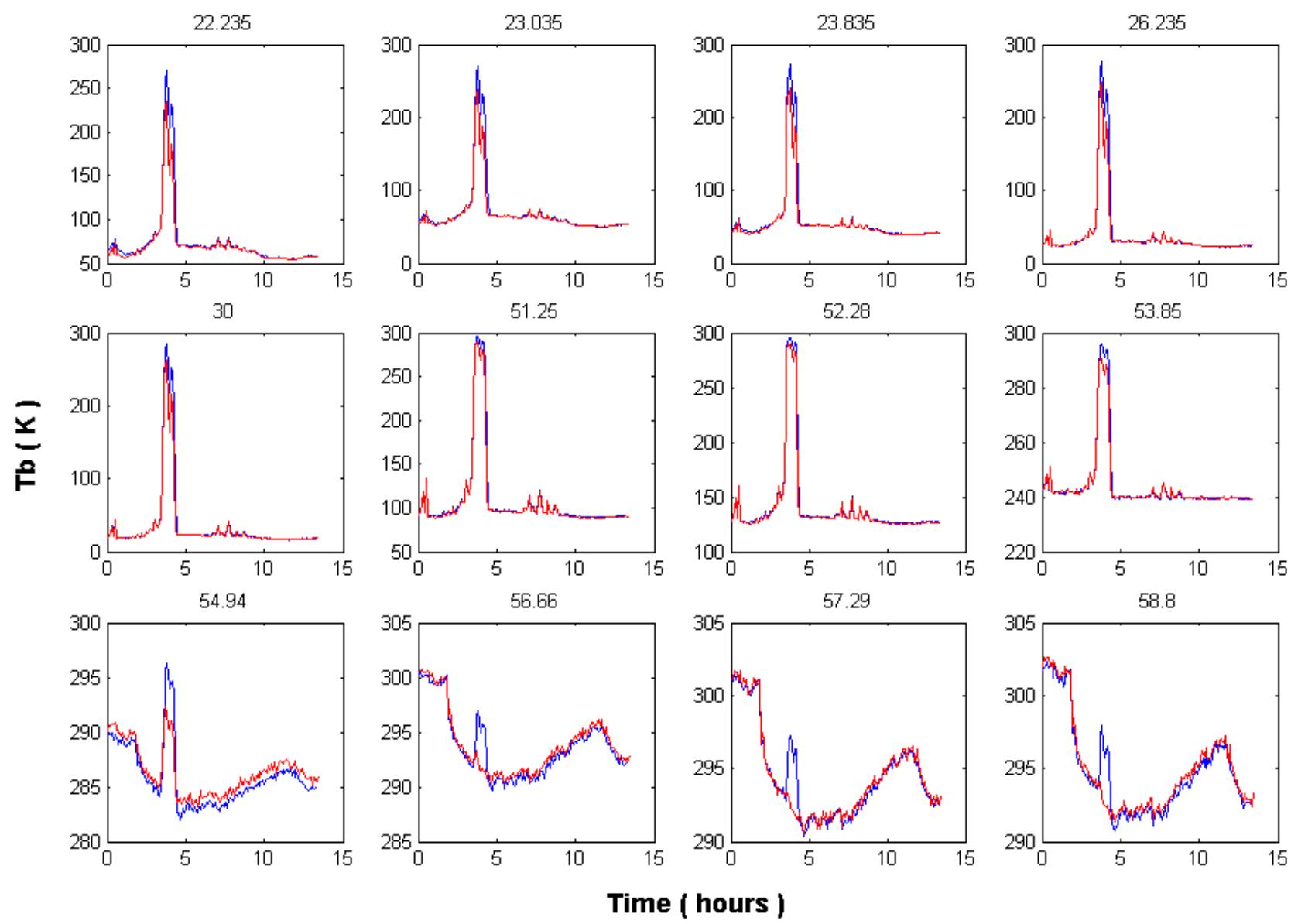

Fig. 7. Same as Fig. 2, but considering two independent co-located MWRP units The only difference between the operational and the development units is a special hydrophobic radome coating mounted on the latter. The operational observations are shown in blue, while observations with the coating are shown in red. The effects of the coating are evident for every channel, reducing Tb during rain. Tb differences range from about $60 \mathrm{~K}$ at $22.2 \mathrm{GHz}$ channel to about $4 \mathrm{~K}$ at $58.8 \mathrm{GHz}$ channel.

\section{References}

D’Auria, G., Marzano, F. S., Pierdicca, N., Pinna Nossai, R., Basili, P., and Ciotti, P.: Remotely sensing cloud properties from microwave radiometric observations by using a modeled cloud data base, Radio Sci., 33, 369-392, 1998.

Jacobson, M. D., Hogg, D. C., and Snider, J. B.: Wet reflectors in millimeter-wave radiometry-Experiment and theory, Trans. Geosci. Remote Sensing, GE-24, 784-791, 1986.

Liu, G. R., Liu, C. C., and Kuo, T. H.: Rainfall intensity estimation by ground-based dual-frequency microwave radiometers, J. Appl. Meteor., 40, 1035-1041, 2001.

Marzano, F. S., Fionda, E., and Ciotti, P.: Simulation of radiometric and attenuation measurements along earth-satellite links in the 10 to $50 \mathrm{GHz}$ band through horizontally-finite convective raincells, Radio Sci., 34, 841-858, 1999.
Marzano, F. S., Fionda, E., Ciotti, P., and Martellucci, A.: Groundbased multi-frequency microwave radiometry for rainfall remote sensing, Trans. Geosci. Rem. Sens., 40, 742-759, 2002.

Tsang, L., Kong, J. A., and Shin, R. T.: Theory of Microwave Remote Sensing, Wiley, New York, 1985.

Smith, E. A., Bauer, P., Marzano, F. S., Kummerow, C. D., McKague, D., Mugnai, A., and Panegrossi, G.: Intercomparison of microwave radiative transfer models for precipitating clouds, Trans. Geosci. Remote Sens., 40, 197-203, 2002.

Ware, R., Solheim, F., Carpenter, R., Gueldner, J., Liljegren, J., Nehrkorn, T., and Vandenberghe, F.: A multi-channel radiometric profiler of temperature, humidity and cloud liquid, Radio Sci., 38(4), 8079, 1-13, 2003.

Westwater, E. R.: Ground-based microwave remote sensing of meteorological variables, in: Atmospheric Remote Sensing by Microwave Radiometry, edited by: Janssen, M. A., Wiley, New York, 1993. 